\title{
Scattering from ramified polymeric systems
}

\author{
M.Benhamou*1, N.Ghaouar ${ }^{1,2}$, A.Gharbi ${ }^{2}$, M.Benmouna ${ }^{3}$ \\ 1 Laboratoire de Physique des Polymères et Phénomènes Critiques, \\ Faculté des Sciences Ben M'sik, B.P. 7955, Casablanca, Morocco \\ 2 Laboratoire de Physique de La Matière Molle, Faculté des Sciences \\ de Tunis, Tunisia \\ 3 Max Planck Institute für Polymerforschung Postfach 3148, \\ D-55021, Mainz, Germany
}

Received August 4, 2003, in final form January 30, 2004

\begin{abstract}
Here, of great interest to us is a quantitative study of the scattering properties from ramified polymeric systems of arbitrary topology. We consider three types of systems, namely ramified polymers in solution, ramified polymer blends, or ternary mixtures made of two ramified polymers of different chemical nature immersed in a good solvent. To achieve the goal of the study, use is made of the Random Phase Approximation. First we determine the exact expression of the form factor of an ideal ramified polymer of any topology, from which we extract the exact expression of its gyration radius. Using the classical Zimm's formulae and the exact form factor, we determine all scattering properties of these three types of ramified polymeric systems. The main conclusion is that ramification of the chains induces drastic changes of the scattering properties.
\end{abstract}

Key words: colloids, macromolecular and polymer solutions, polymer melts, swelling, solubility, segregation, mixing phase separation

PACS: 82.70.Dd, 61.25. Hq, 64.75. $+\mathrm{g}$

\section{Introduction}

The physical system we consider is a ramified polymer of arbitrary topology. By ramified polymer we mean a network made of connected flexible long chains of the same polymerization degree, and with no loops. We assume that the connected chains are chemically identical. As a typical example, we can quote star-polymers [1-3], which are made of the chains tied together at some reticulation point.

In this paper we are interested in the investigation of scattering properties of ramified polymers of any topology, which can be measured using $X$-rays, light or

*Author for correspondence; e-mail: m.benhamou@univh2m.ac.ma 
neutron scattering techniques $[4,5]$. To accomplish this, we will use a simple theoretical approach, which is the so-called Random Phase Approximation (RPA). This method was first suggested by Bohm, Pines and Nozières in the context of strongly interacting systems [6]. Its first extension to polymers was due to de Gennes [4].

It was demonstrated that the RPA approach (mean-field theory) is reliable only for high monomer concentrations or extremely long polymer chains $[4,7,8]$. This means that, in these limits, the asymptotic region where a non-classical asymptotic (or critical) behavior is expected, is very narrow, and hence the mean-field theory can be applied. But, the RPA provided a good tool for describing the critical phase behavior of binary mixtures made of two chemically incompatible linear polymers (of high-molecular weight) [4]. To summarize, we can say that the RPA works only for those polymeric systems in which fluctuations of density (or composition) are too weak. To go beyond this approach, and in order to get a correct asymptotic behavior near criticality where these fluctuations are strong enough, it would be interesting to use the Renormalization-Group ( $\mathrm{RG}$ ) techniques $[9,10]$. The RG approach has been successfully achieved for linear polymers ${ }^{1}[5,7,8,11]$ and recently for star-polymers [12-20]. Concerning these last systems, most of works have focused on the investigation of the configurational properties associated with star-polymers in dilute solution. Especially, the configuration exponent has been computed by several theoretical techniques, from which the contact exponent coinciding with the amplitude of the effective force between adjacent star-polymers has been deduced. For arbitrary topology, however, the problem seems to be more difficult to handle using RG.

Scattering properties are usually investigated through the knowledge of the structure factor, which is directly accessible by scattering experiments. Within the framework of RPA, this structure factor is given by the classical Zimm's formula based on the single contact approximation [21], and which involves the form factor and the interaction parameters.

Our findings are as follows. First, we derive the exact expression of the form factor of an isolated ideal ramified polymer of arbitrary topology, and study all its analytical properties. From the obtained form factor, we deduce the exact expression of the corresponding gyration radius. Second, we determine the expression of the structure factor of ramified polymers in solution, through Zimm's formula. To illustrate our general results, we consider a star-polymer, and compute the corresponding structure factor, as a function of the wave-vector and parameters of the problem, which are the number of chains inside the star-polymers, the polymer concentration and the excluded volume parameter. Third, we demonstrate that the structure factor in the solution of ramified polymers of a given topology is more important than that of a linear chain having the same number of monomers. This means that the presence of reticulation points has a tendency to reduce the fluctuations of monomer concentration. Fourth, we consider a blend of two chemically different ramified polymers of arbitrary topology. Using RPA, we investigate all critical properties of the mixture. Finally, we extend the study to ternary mixtures made of two incompatible ramified

\footnotetext{
${ }^{1}$ An extensive list of works on the subject can be found in references [7] and [8].
} 
polymers immersed in a good solvent. More precisely, we compute the total structure factor describing the space correlations of the local total polymer concentration. The main result is that the presence of the chain ramification yields a drastic change of the scattering properties of the systems under investigation.

The remaining presentation proceeds as follows. In section 2, we exactly compute the form factor of an isolated ideal polymer network of arbitrary topology, and deduce its gyration radius. Section 3 is devoted to the determination of the structure factor of ramified polymers in a solution. In sections 4 and 5, we study the scattering properties from ramified polymer blends and ternary solutions, respectively. We draw our conclusions in the last section. Some technical details are relegated to Appendix.

\section{The exact form factor}

Consider an ideal ramified polymer (network) $G$ made of $f$ identical Brownian chains linked together to some reticulation points or vertices (figure 1), with the constraint that no loop is present. A free extremity of a chain can be regarded as a one-leg vertex.

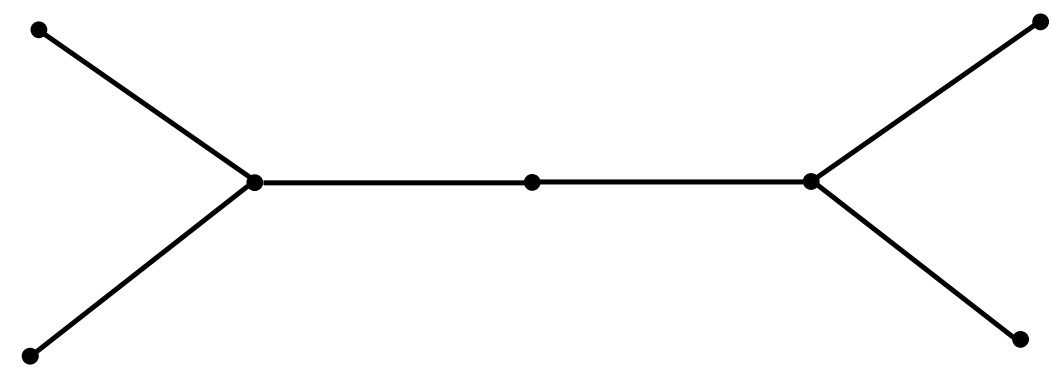

Figure 1. A polymer network made of $f=6$ identical chains.

The $f$ chains of $G(a=1, \ldots, f)$ can be described within the framework of the standard continuous model $[5,13,16,19,22]$. The Brownian statistical weight reads

$$
\mathcal{P}_{0}\{\mathbf{r}\}=\exp \left\{-\frac{1}{2} \sum_{a=1}^{f} \int_{0}^{S} \mathrm{~d} s\left(\frac{\mathrm{d} \mathbf{r}_{a}}{\mathrm{~d} s}\right)^{2}\right\} .
$$

Here, $\mathbf{r}_{a}(s)$ is the configuration of the chain $a$ in the $d$-dimensional euclidean space $\mathbf{R}^{d}$, and the coordinate $s \in[0, S]$ marks a point along this chain. In equation (1), $S$ represents the Brownian area defining the mean squared end-to-end distance of a free chain, that is $R^{2}=d \times S$. In fact, the phenomenological parameter $S$ can be related to the polymerization degree $N$ of real chains by: $S=N \sigma^{2} / d[5]$, where $\sigma$ is the monomer size (Kuhn statistical unit).

With the help of the above statistical weight, one can calculate the mean-value $\langle F\rangle$ of any functional $F$ of configurations $\left\{\mathbf{r}_{a}(s), a=1, \ldots, f ; 0 \leqslant s \leqslant S\right\}$, which is defined as usual by $[13,16,19]$

$$
\langle F\rangle=\frac{1}{Z_{\mathrm{G}}(f \times S)} \cdot \frac{\int \mathrm{d}\{\mathbf{r}\} \delta_{d}(G) F\{\mathbf{r}\} \mathcal{P}_{0}\{\mathbf{r}\}}{\int \mathrm{d}\{\mathbf{r}\} \mathcal{P}_{0}\{\mathbf{r}\} \prod_{a=1}^{\mathcal{N}} \delta_{d}\left[\mathbf{r}_{a}(0)\right]},
$$


where

$$
Z_{\mathrm{G}}(f \times S)=\frac{\int \mathrm{d}\{\mathbf{r}\} \delta_{d}(G) \mathcal{P}_{0}\{\mathbf{r}\}}{\int \mathrm{d}\{\mathbf{r}\} \mathcal{P}_{0}\{\mathbf{r}\} \prod_{a=1}^{\mathcal{N}} \delta_{d}\left[\mathbf{r}_{a}(0)\right]}
$$

accounts for the partition function of the polymer network $G$. There, the notation $\delta_{d}(G)$ is symbolic; it is the product of all necessary Dirac distributions $\delta_{d}$ 's in direct space connecting the chains in the polymer network $G$, plus one for fixing the origin. Of course, the above definition is independent of the choice of the fixed vertex in space.

We are interested in determining the form factor of a polymer network $G$, which is defined by the mean-value

$$
P_{\mathrm{G}}(q)=\frac{1}{f^{2} S^{2}}\left\langle\sum_{a=1}^{f} \sum_{b=1}^{f} \int_{0}^{S} \mathrm{~d} s_{a} \int_{0}^{S} \mathrm{~d} s_{b} \exp \left\{\mathbf{i q}\left[\mathbf{r}_{a}\left(s_{a}\right)-\mathbf{r}_{b}\left(s_{b}\right)\right]\right\}\right\rangle .
$$

Here, $s_{a} \in[0, S]$ and $s_{b} \in[0, S]$ are the coordinates of the points belonging to the chains $a$ and $b$, respectively. As usual, the form factor depends only on the module $q=|\mathbf{q}|$ of the wave-vector $\mathbf{q}$.

From its definition, the form factor can be decomposed as follows:

$$
P_{\mathrm{G}}(q)=P_{11}(q)+P_{12}(q),
$$

where $P_{11}(q)$ and $P_{12}(q)$ represent the intra- and inter-chain contributions, respectively. We find that the intra-chain form factor is directly proportional to that of a single ideal chain, that is

$$
P_{11}(q)=\frac{1}{f} g_{\mathrm{D}}(u)
$$

where

$$
g_{\mathrm{D}}(u)=\frac{2}{u^{2}}\left(u-1+\mathrm{e}^{-u}\right)
$$

is the standard Debye function [4,5], with the notation $u=(3 / d) q^{2} R_{0}^{2}$, where $R_{0}^{2}=$ $\sigma^{2} N / 6$ is the squared gyration radius of a single ideal chain.

The inter-chain form factor is formally defined by the mean-value

$$
P_{12}(q)=\frac{2}{f^{2} S^{2}}\left\langle\sum_{a>b} \int_{0}^{S} \mathrm{~d} s_{a} \int_{0}^{S} \mathrm{~d} s_{b} \exp \left\{\mathbf{i q}\left[\mathbf{r}_{a}\left(s_{a}\right)-\mathbf{r}_{b}\left(s_{b}\right)\right]\right\}\right\rangle .
$$

The computation of $P_{12}(q)$, which is new, merits a particular attention.

To do the calculations, we first consider a pair of chains $(a, b)$. Since there are no loops, there exists only one connected path, $\gamma$, connecting the chain $a$ to the chain $b$ in the network $G$. We denote by $|\gamma|$ the number of the chains belonging to $\gamma$. We find that the contribution of the pair $(a, b)$ is

$$
\begin{gathered}
\left\langle\int_{0}^{S} \mathrm{~d} s_{a} \int_{0}^{S} \mathrm{~d} s_{b} \exp \left\{\mathbf{i q}\left[\mathbf{r}_{a}\left(s_{a}\right)-\mathbf{r}_{b}\left(s_{b}\right)\right]\right\}\right\rangle=\mathrm{e}^{-|\gamma| S q^{2} / 2} \times \\
\left(\int_{0}^{S} \mathrm{~d} s \mathrm{e}^{-(S-s) q^{2} / 2}\right)\left(\int_{0}^{S} \mathrm{~d} s^{\prime} \mathrm{e}^{-s^{\prime} q^{2} / 2}\right) .
\end{gathered}
$$


In this equality, the $\exp \left\{-|\gamma| S q^{2} / 2\right\}$-factor is the contribution of chains belonging to the path $\gamma$; each contributes with the same $\exp \left\{-S q^{2} / 2\right\}$-factor. The remaining two trivial factors in the right-hand side of equation (7) are the contributions of the chains $a$ and $b$, where the wave-vector $\mathbf{q}$ is inserted into the former and its opposite $\mathbf{- q}$ into the second (figure 2). The mixing form factor can be obtained summing over all pairs of distinct chains. This is equivalent to the sum over all connected paths $\gamma$ 's of the polymer network $G$, with $|\gamma| \geqslant 2$. We find that

$$
P_{12}(q)=\frac{2}{f^{2}}\left(\frac{\mathrm{e}^{u}-1}{u}\right)^{2} \sum_{\{\gamma \subset G ;|\gamma| \geqslant 2\}} \mathrm{e}^{-|\gamma| u} .
$$

The notation $\gamma \subset G$ means that $\gamma$ is a connected path between the nodes of the polymer network $G$.

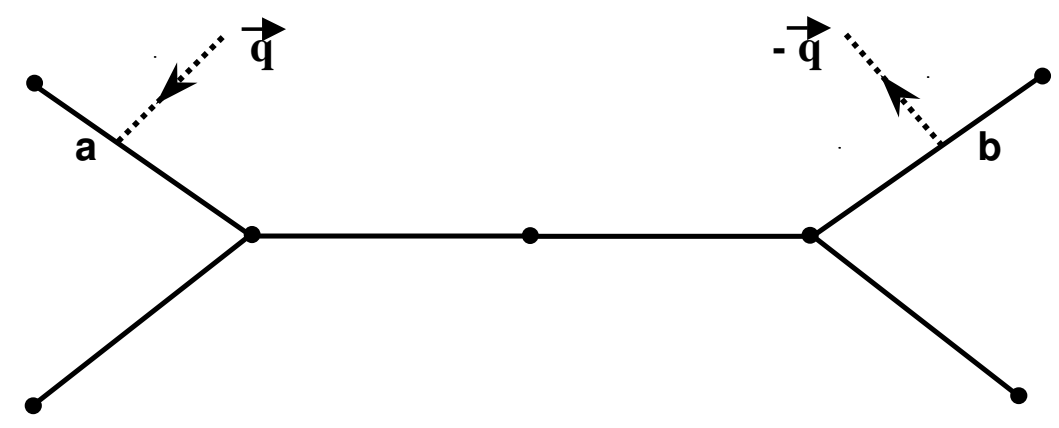

Figure 2. A polymer network made of $f=6$ identical chains, with insertion of the wave-vector $\mathbf{q}$.

The final result is obtained combining relations (5) and (8)

$$
P_{\mathrm{G}}(q)=\frac{1}{f}\left[g_{\mathrm{D}}(u)+\frac{2}{f}\left(\frac{\mathrm{e}^{u}-1}{u}\right)^{2} \sum_{\{\gamma \subset G ;|\gamma| \geqslant 2\}} \mathrm{e}^{-|\gamma| u}\right],
$$

where $g_{\mathrm{D}}(u)$ is the Debye function defined by relation $(5 b)$. We will set

$$
h_{\mathrm{G}}(u)=\sum_{\{\gamma \subset G ;|\gamma| \geqslant 2\}} \mathrm{e}^{-|\gamma| u},
$$

which is a function depending on the topological structure of the considered network.

The exact equation (9) giving the form factor of any ideal polymer network, calls for some commentaries.

Firstly, it generalizes the Debye function describing the form factor of a single linear chain.

Secondly, we show that this form factor is an analytic function around $q^{2}=0$ (or equivalently around $u=0$ ).

Thirdly, at a high wave-vector (in neutron-scattering), we find that the form factor scales as: $P_{\mathrm{G}}(q) \simeq\left(4 d / f N \sigma^{2}\right) q^{-2}(q \rightarrow+\infty)$, independently of the particular 
topological structure of the polymer network $G$. Indeed, at small-scales, one does not feel the presence of reticulation points connecting the chains in the network $G$. In addition, this asymptotic behavior tells us that an ideal polymer network is a fractal object having the same Hausdorff dimension [5] as a single linear chain, which is 2 .

Finally, for $f=1$, we recover the known result that the form factor of a single polymer chain is given by the Debye function.

Now, to illustrate the generalized formula (9), we consider two particular polymer networks, which are a star-polymer made of $f$ linear chains tied together to some vertex and a linear network, $L$, which is presented as a succession of $f$ connected linear chains of polymerization degree $N$. To determine the corresponding form factors, we will need to specify the expression of the topological function $h_{\mathrm{G}}(u)$. For a star-polymer with $f \geqslant 2$, all paths $\gamma$ 's contain only two chains, and thus, $|\gamma|=2$. Their number is exactly $f(f-1) / 2$, and the corresponding topological function reads

$$
h_{\text {star }}(u)=\frac{f(f-1)}{2} \mathrm{e}^{-2 u} .
$$

Then, the form factor of an isolated star-polymer is

$$
P_{\text {star }}(q)=\frac{f-3+2 u+2(2-f) \mathrm{e}^{-u}+(f-1) \mathrm{e}^{-2 u}}{f u^{2}} .
$$

From this expression, we recover the form factor of a single chain in the limit $f \rightarrow 1$. For a linear network $L$, we find

$$
\begin{aligned}
h_{\text {linear }}(u) & =\sum_{\{\gamma \subset L ;|\gamma| \geqslant 2\}} \mathrm{e}^{-|\gamma| u} \\
& =\sum_{n=2}^{f}(f-n+1) \mathrm{e}^{-n u}=\frac{f-1-f \mathrm{e}^{-u}+\mathrm{e}^{-u f}}{\left(\mathrm{e}^{u}-1\right)^{2}} .
\end{aligned}
$$

Having inserted this formula into equation (9) we get the form factor

$$
P_{\text {linear }}(q)=g_{\mathrm{D}}(w)
$$

where $g_{\mathrm{D}}(w)$ is the Debye function of argument $w=f u$. Thus, we recover, as it should be, the form factor of a single linear chain having the same number of monomers, that is $f N$, as the linear network.

Incidentally, the form factor of any polymer network $G$ is smaller than that of a star-polymer having the same number of chains. More precisely, we have

$$
P_{\mathrm{G}}(q) \leqslant P_{\text {star }}(q) \text {. }
$$

This inequality is independent of the particular topological structure of the polymer network $G$. As a matter of fact, this inequality is a direct consequence of the obvious result according to which $h_{\mathrm{G}}(u) \leqslant h_{\text {star }}(u)$. 
On the other hand, we demonstrate in Appendix the important inequality

$$
P_{\text {linear }}(q) \leqslant P_{\mathrm{G}}(q),
$$

which states that the form factor of any polymer network made of $f$ chains of polymerization degree $N$ is greater than that of a linear chain having the same number of monomers, that is $f N$. The above inequality occurs for all values of the wave-vector and for any topology. The physical sense of inequalities (13a) and (13b) will be discussed bellow.

The extended formula (9) for the form factor enables us to compute the exact expression of the gyration radius of an ideal polymer network of any topology.

Denote by $R_{\mathrm{G}}^{2}$, the squared gyration radius of an ideal polymer network $G$ of arbitrary topology. It is defined by the following mean-value

$$
R_{\mathrm{G}}^{2}=\frac{1}{2 f^{2} S^{2}}\left\langle\sum_{a=1}^{f} \sum_{b=1}^{f} \int_{0}^{S} \mathrm{~d} s_{a} \int_{0}^{S} \mathrm{~d} s_{b}\left[\mathbf{r}_{a}\left(s_{a}\right)-\mathbf{r}_{b}\left(s_{b}\right)\right]^{2}\right\rangle .
$$

We note that the quantity $R_{\mathrm{G}}^{2}$ can be obtained expanding the form factor with respect to the variable $q^{2}$, that is [23]

$$
P_{\mathrm{G}}(q)=1-\frac{q^{2} R_{\mathrm{G}}^{2}}{d}+\cdots, \quad \text { (Guinier regime) }
$$

Expanding the generalized formula (9) to the second order around $q^{2}=0$ (or equivalently around $u=0$ ) yields the exact expression for the gyration radius

$$
R_{\mathrm{G}}^{2}=\kappa_{\mathrm{G}} \frac{\sigma^{2}}{6} N
$$

with the topological factor

$$
\kappa_{\mathrm{G}}=\frac{1}{f}\left(1+6 \frac{J-I}{f}\right)
$$

where

$$
I=\sum_{\{\gamma \subset G ;|\gamma| \geqslant 2\}} 1, \quad J=\sum_{\{\gamma \subset G ;|\gamma| \geqslant 2\}}|\gamma|
$$

are two quantities depending on the topology of the polymer network $G$. The former simply represents the number of all possible connected paths within $G$ containing at least two chains. The second factor is the total length of all connected paths $\gamma$ 's, with $|\gamma| \geqslant 2$. In addition, we find that the factor $I$ is independent of the particular topological structure of the polymer network $G$. More precisely, we show for any polymer network the formula

$$
I=\frac{f(f-1)}{2}
$$

This is compatible with the zero-scattering angle limit of the form factor: $P_{\mathrm{G}}(q=$ $0)=1$, which is a direct consequence of its definition (3). The above expression tells 
us that two polymer networks of the same number of chains correspond to the same factor $I$. To demonstrate the above result, we note that to each pair of chains, there is only one path connecting them in the network. Thus, the total number of paths containing at least two chains identifies with the number of distinct pairs of chains, which is precisely $f(f-1) / 2$. The second factor $J$, however, crucially depends on the topological nature of the considered network. This same factor is bounded from below, that is

$$
J=\sum_{\{\gamma \subset G ;|\gamma| \geqslant 2\}}|\gamma| \geqslant 2 I .
$$

We have used the fact that $|\gamma| \geqslant 2$, for all connected paths $\gamma$ 's. The above inequality implies that the topological factor $\kappa_{\mathrm{G}}$ satisfies the inequality

$$
\kappa_{\mathrm{G}} \geqslant \frac{3 f-2}{f} \text {. }
$$

Hence, we have

$$
R_{\mathrm{G}}^{2} \geqslant R_{\mathrm{star}}^{2}
$$

where $R_{\text {star }}^{2}$ is the squared gyration radius of an ideal star-polymer

$$
R_{\mathrm{star}}^{2}=\frac{3 f-2}{f} \frac{\sigma^{2}}{6} N .
$$

We emphasize that the same result has been derived directly in the classical textbook by Yamakawa [24]. For $f=1$, we recover the squared gyration radius of a single chain, that is $\sigma^{2} N / 6$.

The knowledge of the form factor will enable us to write an explicit expression for the structure factors of identical ramified polymers in a solution, a ramified polymer blend or a ternary mixture made of two incompatible ramified polymers in a good solvent. This will be done within the framework of RPA.

\section{Scattering from ramified polymers in a solution}

In the RPA spirit, the structure factor is given by the Zimm's formula $[22,26,27]$ according to which

$$
S_{\mathrm{G}}^{-1}(q)=S_{0}^{-1}(q)+v
$$

where

$$
S_{0}(q)=\phi f N P_{\mathrm{G}}(q)
$$

is the bare structure factor, and $v$ represents the dimensionless excluded volume parameter defined by

$$
v=\left(\frac{1}{\phi_{\mathrm{S}}}-2 \chi_{\mathrm{PS}}\right)
$$

Here, $\phi_{\mathrm{S}}$ represents the volume fraction of solvent and $\chi_{\mathrm{PS}}$ is the polymer-solvent Flory-Huggins interaction parameter. Explicitly, the structure factor is formally given by

$$
S_{\mathrm{G}}^{-1}(q)=\frac{1}{\phi f N P_{\mathrm{G}}(q)}+v
$$


where $P_{\mathrm{G}}(q)$ is the form factor defined by relation (9), and $\phi$ accounts for the monomer volume fraction related to $\phi_{\mathrm{S}}$ by the incompressibility condition: $\phi+\phi_{\mathrm{S}}=1$. Equation (21) tells us that all the dependence on topology is contained in the form factor $P_{\mathrm{G}}(q)$.

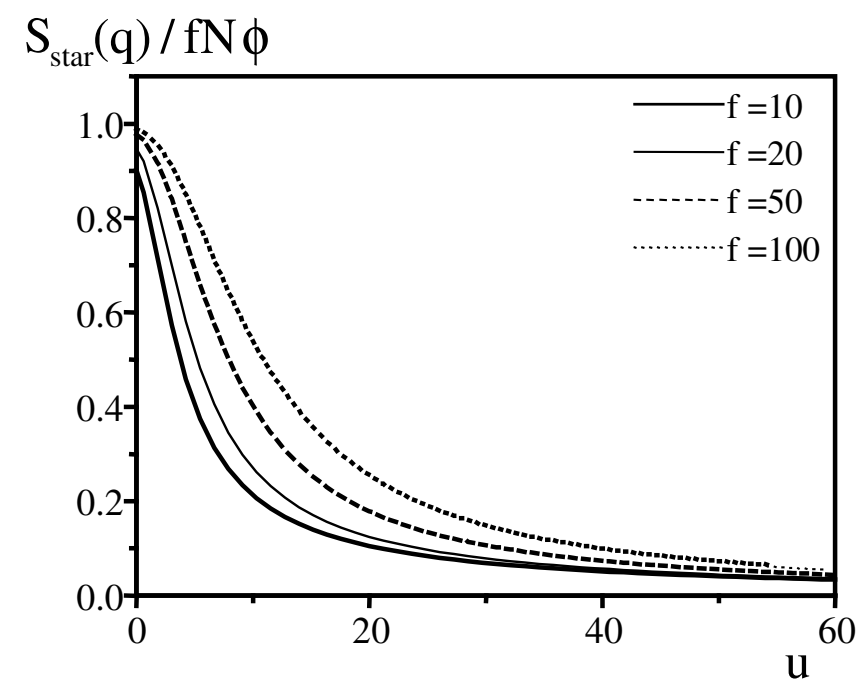

Figure 3. Superposition of the structure factors (reduced by the $\phi N$-factor) of star-polymers in a solution, versus the dimensionless variable $u=(3 / d) q^{2} R_{0}^{2}$, for 4 values of the number of branches $f$, that is $f=10,20,50,100$, with parameter $v \phi N=1$.

For star-polymers in solution, for instance, the structure factor, $S_{\text {star }}(q)$, is given by equation (21), where the form factor, $P_{\text {star }}(q)$, is that defined by the exact formula $(11 b)$. In fact, as it should be, the structure factor $S_{\text {star }}(q)$ is analytic in the $u$ variable, and decreases with the increasing wave-vector. At a fixed wave-vector ( $u$ is fixed) and monomer concentration, this structure factor increases with the increasing $f$. To illustrate this tendency, we superpose, in figure 3 , the variations of the reduced structure factor $S_{\text {star }}(q) / \phi N$ of a star-polymer versus the dimensionless variable $u=(3 / d) q^{2} R_{0}^{2}\left(R_{0}^{2}=\sigma^{2} N / 6\right)$, for 4 values of $f$, that is $f=10,20,50,100$, with parameter $v \phi N=1$. In figure 4, we superpose the variations of the structure factor (reduced by the $\phi f N$-factor) of star-polymers in a solution and its linear homologous versus $u$, with parameters $f=10$ and $v f \phi N=10$. The curve relative to starpolymers remains above that of the linear ones, for all values of $u$-variable and parameters of the problem.

Finally, we note that inequalities $(13 a)$ and (13b) together with expression (21) imply that the structure factor $S_{\mathrm{G}}(q)$ of any polymer network $G$ is such that

$$
S_{\text {linear }}(q) \leqslant S_{\mathrm{G}}(q) \leqslant S_{\text {star }}(q) \text {. }
$$

This means that the structure factor of ramified polymers of any topology is always bounded from below by that of linear ones and from above by that of star-polymers. 


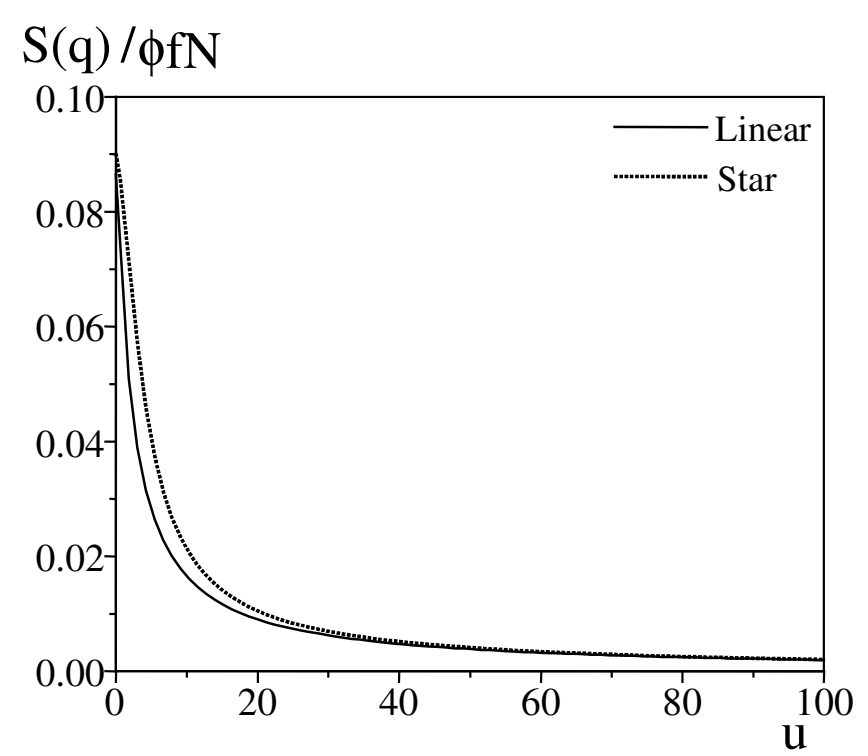

Figure 4. Superposition of the structure factor (reduced by the $\phi f N$-factor) of star-polymers in a solution and that of its linear homologous, versus the dimensionless variable $u=(3 / d) q^{2} R_{0}^{2}$, with parameters: $f=10, v \phi f N=10$.

The physical sense of these inequalities can be understood as follows. Inside a ramified polymer, the fluctuations of monomer concentration are more important than inside a star-polymer, and less important than inside a linear polymer network. The above inequalities are valid for all values of the wave-vector and parameters of the problem.

\section{Scattering from ramified polymer blends}

Now, consider a mixture made of two ramified polymers $A$ and $B$ of different chemical nature. We denote by $f_{\alpha}(\alpha=A, B)$, the number of chains inside the polymer network $\alpha=A, B$, and by $N_{\alpha}$, their common polymerization degree. We denote by

$$
S_{\alpha \beta}(q)=\sigma^{-d} \int \mathrm{d}^{d} r G_{\alpha \beta}(r) \mathrm{e}^{\mathrm{iqr}}, \quad(\alpha, \beta=A, B),
$$

the partial structure factor between species $\alpha$ and $\beta$. It is the Fourier transform of the composition correlation function

$$
G_{\alpha \beta}(r)=\left\langle\phi_{\alpha}(0) \phi_{\beta}(\mathbf{r})\right\rangle-\left\langle\phi_{\alpha}(0)\right\rangle\left\langle\phi_{\beta}(\mathbf{r})\right\rangle, \quad(\alpha, \beta=A, B),
$$

where $\phi_{\alpha}$ stands for the composition of polymer $\alpha$.

We assume that the considered mixture is incompressible. That is, it satisfies the incompressibility condition: $\phi_{A}+\phi_{B}=1$. This condition implies that the three partial structure factors are not independent of each other, and we manifestly have

$$
S_{A A}(q)=S_{B B}(q)=-S_{A B}(q) .
$$


We will use the notation: $S(q) \equiv S_{A A}(q)$.

Within the framework of RPA, the desired structure factor obeys the equality [4]

$$
S^{-1}(q)=S_{A 0}^{-1}(q)+S_{B 0}^{-1}(q)-2 \chi,
$$

where $S_{\alpha 0}(q)=f_{\alpha} N_{\alpha} \phi_{\alpha} P_{G_{\alpha}}(q)$ is the bare structure factor of species $\alpha=A, B$, and $\chi \equiv \chi_{A B}$ is the standard Flory interaction parameter [4,27], which describes the chemical interaction between the two components. Here, $P_{G_{\alpha}}(q)$ is the form factor of an ideal ramified polymer $\alpha$ given by equation (9). Expression (24) shows that all the dependence on topology is entirely contained in the form factors $P_{G_{A}}(q)$ and $P_{G_{B}}(q)$.

With the help of the above expression, we can deduce all critical properties of the mixture, in particular, the spinodal curve along which the isotherm compressibility diverges. In fact, this latter is directly proportional to the zero-scattering angle limit of the structure factor. The equation defining this curve in the $(\phi, \chi)$-plane can be obtained equating $S^{-1}(0)$ to zero. Explicitly, we have

$$
\chi_{\mathrm{c}}=\frac{1}{2 f_{A} N_{A} \phi}+\frac{1}{2 f_{B} N_{B}(1-\phi)},
$$

where $\phi$ represents the composition of $A$-polymer. The above formula shows that the location of the spinodal curve is independent of the particular topological structure of the two networks $G_{A}$ and $G_{B}$. The only dependence comes from the explicit appearance of their respective numbers of chains $f_{A}$ and $f_{B}$. In comparison with a mixture of two linear polymers of polymerization degrees $N_{A}$ and $N_{B}$, relation (25) clearly shows that the topology tends to reduce the compatibility domain. This is due to the fact that at least one of the numbers of chains $f_{A}$ and $f_{B}$ is greater than 1.

For a symmetric mixture, that is when the two ramified polymers have the same topology, and

$$
f_{A}=f_{B} \equiv f, \quad N_{A}=N_{B} \equiv N, \quad P_{G_{A}}(q)=P_{G_{B}}(q) \equiv P_{\mathrm{G}}(q),
$$

the corresponding structure factor takes the following simplified expression

$$
S_{\mathrm{G}}^{-1}(q)=2 \chi_{\mathrm{c}} P_{\mathrm{G}}^{-1}(q)-2 \chi .
$$

We have used the definition of the critical Flory interaction parameter $\chi_{\mathrm{c}}$.

As an illustration, we superpose, in figure 5, the variations of the structure factor of a mixture of identical star-polymers versus the $u$-variable, for 4 values of the number of branches, that is $f=10,20,50,100$. These curves are drawn with parameters $\chi_{\mathrm{c}}=2 \times 10^{-3}$ and $\chi=0.5 \times 10^{-4}<\chi_{\mathrm{c}}$ (one-phase region). They reflect the natural increase of the structure factor, as a function of the number of branches, at fixed $u$-variable and parameters of the problem. We compare, in figure 6 , the structure factor of a binary star-polymer mixture and that of its linear homologous. This curve shows that the star-polymer structure factor remains above the linear one. 


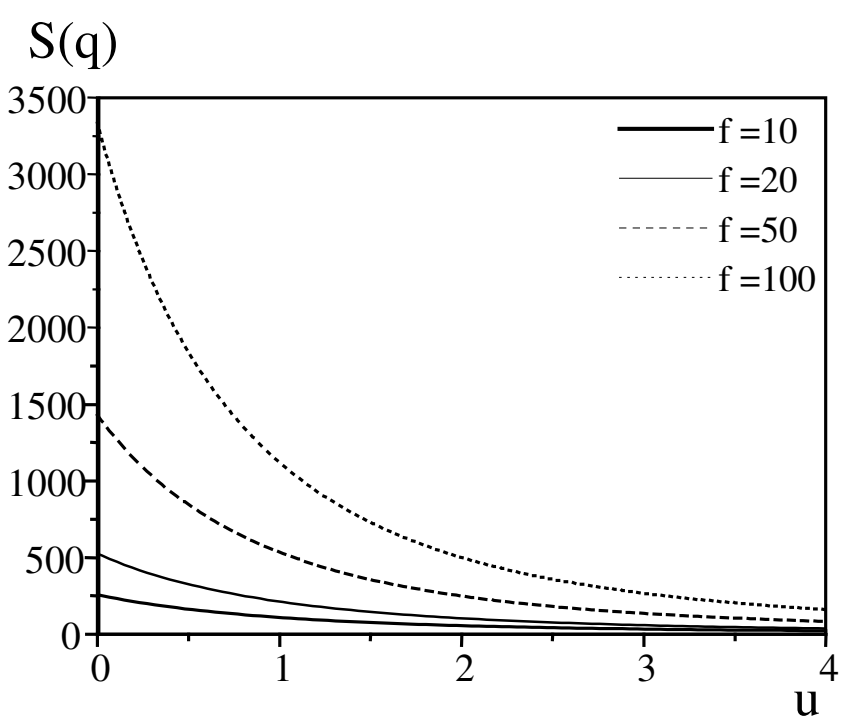

Figure 5. Superposition of the structure factors of a symmetric binary mixture of two incompatible star-polymers, versus the $u$-variable, for 4 values of the number of branches, that is $f=10,20,50,100$. These curves are drawn with parameters: $N=100, \chi=10^{-3}$.

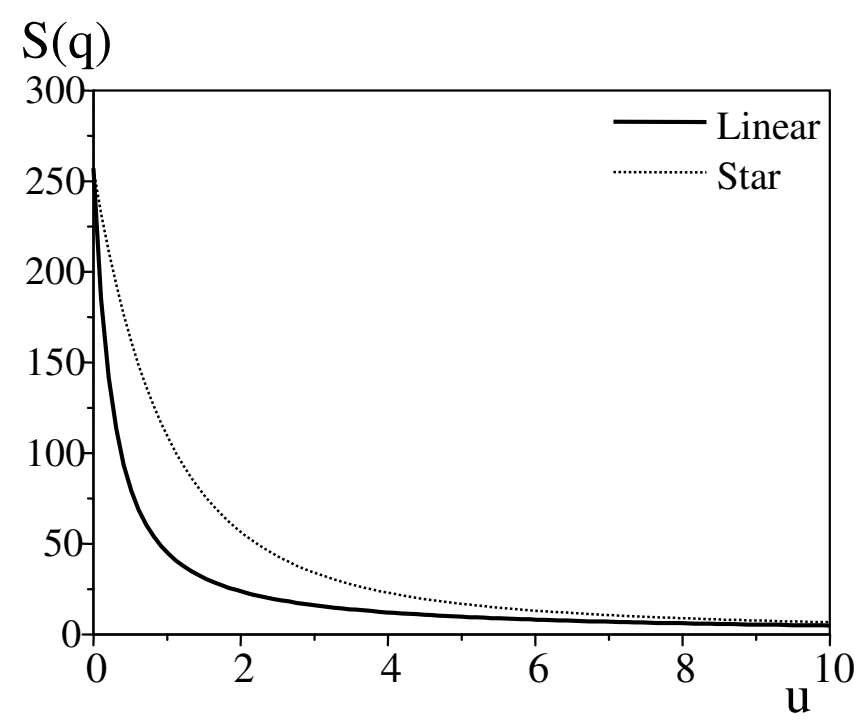

Figure 6. Curves representing a comparison between the structure factor of a symmetric binary mixture of two incompatible star-polymers and that of its linear homologous, with $f=10$. These curves are drawn with parameters: $\chi_{\mathrm{c}}=2 \times 10^{-3}$, $\chi=0.5 \times 10^{-3}<\chi_{\mathrm{c}}$. 
Finally, as for the polymer in a solution case, we show that the structure factor is always bounded from below by that of a linear network and from above by that of a star-polymer. In fact, this originates from a reduction of the composition fluctuations due to the presence of chain ramifications (there is at least one chain attached to the others by its two extremities).

\section{Scattering from ternary mixtures}

Now, consider a ternary mixture made of two chemically different ramified polymers $A$ and $B$ in a good solvent. We note that when a good solvent is added to a polymer blend, the chemical compatibility of the mixture is substantially enhanced by increasing the solvent concentration. This implies that the critical temperature is decreased due to the increase of entropy of mixing resulting from the presence of a solvent. Our aim is to quantitatively investigate the impact of topology on the critical properties of the system. This will be done using the RPA approach.

We will designate by $\phi_{A}$ and $\phi_{B}$, the volume fractions of the ramified polymers $A$ and $B$, and by $\phi_{\mathrm{S}}$ the solvent one. For an incompressible ternary mixture, we have the natural condition that: $\phi_{A}+\phi_{B}+\phi_{\mathrm{S}}=1$. We will set, for convenience, $\phi_{A}=x \phi$ and $\phi_{B}=(1-x) \phi$, where $x$ is the composition of species $A$ and $\phi=\phi_{A}+\phi_{B}$ is the overall monomer volume fraction.

We are interested in the computation of the partial structure factors $S_{A A}(q)$, $S_{B B}(q)$ and $S_{A B}(q)=S_{B A}(q)$, as functions of the wave-vector and parameters of the problem. According to references [25] and [26], these structure factors take the following forms

$$
\begin{aligned}
S_{A A}(q) & =\frac{S_{A}^{0}(q)\left[1+v_{B} S_{B}^{0}(q)\right]}{D(q)} \\
S_{B B}(q) & =\frac{S_{B}^{0}(q)\left[1+v_{A} S_{A}^{0}(q)\right]}{D(q)} \\
S_{A B}(q) & =S_{B A}(q)=-\frac{v_{A B} S_{A}^{0}(q) S_{B}^{0}(q)}{D(q)},
\end{aligned}
$$

where $S_{\alpha}^{0}(q)=f_{\alpha} N_{\alpha} \phi_{\alpha} P_{G_{\alpha}}(q),(\alpha=A, B)$ represents the bare structure factor of species $\alpha$, and

$$
v_{A}=\frac{1}{\phi_{\mathrm{S}}}-2 \chi_{A \mathrm{~S}}, \quad v_{B}=\frac{1}{\phi_{\mathrm{S}}}-2 \chi_{B \mathrm{~S}}
$$

account for the excluded volume parameters relative to polymers $A$ and $B$, where $\chi_{A S}$ and $\chi_{B S}$ are polymer $A$-solvent and polymer $B$-solvent Flory-Huggins interaction parameters. The notation $v_{A B}$ means

$$
v_{A B}=\frac{1}{\phi_{\mathrm{S}}}-\chi_{A \mathrm{~S}}-\chi_{B \mathrm{~S}}+\chi
$$

where $\chi$ is the Flory interaction parameter describing the chemical interactions between unlike polymers. The denominator in expressions (27)-(29) is given by

$$
D(q)=1+v_{A} S_{A}^{0}(q)+v_{B} S_{B}^{0}(q)+\left(v_{A} v_{B}-v_{A B}^{2}\right) S_{A}^{0}(q) S_{B}^{0}(q) .
$$


It is useful to recall the expression of the total structure factor $S_{T}(q)$, which describes the space correlations of the local total polymer concentration

$$
S_{T}(q)=S_{A A}(q)+S_{B B}(q)+2 S_{A B}(q)
$$

Explicitly, we have

$$
S_{T}(q)=\frac{S_{A 0}(q)+S_{B 0}(q)+\left(v_{A}+v_{B}-2 v_{A B}\right) S_{A 0}(q) S_{B 0}(q)}{D(q)},
$$

where the denominator $D(q)$ is given by relation (32), with $S_{A 0}(q)=x f_{A} N_{A} \phi P_{G_{A}}(q)$ and $S_{B 0}(q)=(1-x) f_{B} N_{B} \phi P_{G_{B}}(q)$.

These relations clearly show that the scattering properties from the mixture crucially depend on the topological structure of its two components. This dependence is entirely contained in the bare structure factors appearing in equation (34), through the form factors relative to the two polymers.

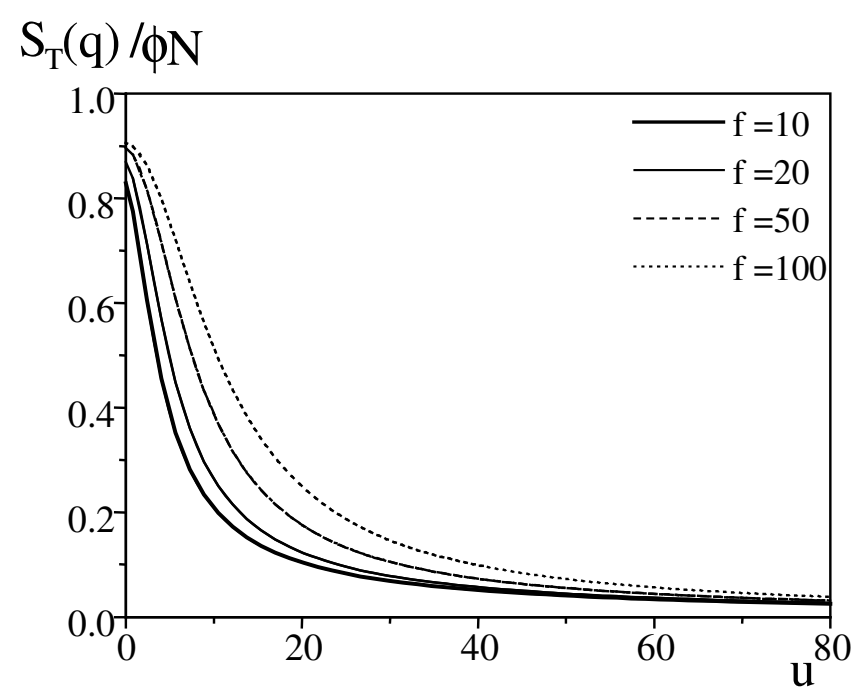

Figure 7. Superposition of the total structure factors (reduced by the $\phi N$-factor) of a symmetric ternary mixture made of two incompatible star-polymers immersed in a good solvent, for 4 values of the number of branches $f$, that is $f=10$, $20,50,100$. These curves are drawn with parameters: $\chi f N \phi=1.8, v N \phi=1$.

In what follows, we will choose symmetric ternary mixtures, that is the two polymers have the same topology, and

$$
\begin{gathered}
f_{A}=f_{B} \equiv f, \quad N_{A}=N_{B} \equiv N, \quad x=1 / 2, \\
P_{G_{A}}(q)=P_{G_{B}}(q) \equiv P_{\mathrm{G}}(q), \quad \chi_{A \mathrm{~S}}=\chi_{B S} \equiv \chi_{\mathrm{PS}},
\end{gathered}
$$

where the common form factor $P_{\mathrm{G}}(q)$ is that given by formula (9). With these considerations, the total structure factor becomes

$$
\frac{S_{T}(q)}{f N \phi}=P_{\mathrm{G}}(q) \frac{4-2 f N \phi \chi P_{\mathrm{G}}(q)}{4+4 v f N \phi P_{\mathrm{G}}(q)+\left[v^{2}-(v+\chi)^{2}\right] f^{2} N^{2} \phi^{2} P_{\mathrm{G}}^{2}(q)} .
$$


As an illustration, we report, in figure 7, a superposition of the variations of the total structure factor of binary star-polymer mixtures, for 4 values of the number of branches $f$, that is $f=10,20,50,100$. As it should be, the total structure factor increases with the increasing $f$, for all values of the wave-vector.



Figure 8. Curves representing the total structure factor (reduced by the $\phi f N$ factor) of a symmetric ternary mixture made of two incompatible star-polymers immersed in a good solvent and that of its linear homologous. These curves are drawn with parameters: $f=10, \chi f N \phi=1.8, v f N \phi=10$.

In figure 8, we draw the curves representing the total structure factor of a starpolymer mixture and that of its linear homologous, with $f=10$. This figure reflects the reduction of the total structure factor due to the presence of ramifications.

Now, the spinodal equation defining the condition under which a phase separation occurs, is as follows

$$
D(q=0)=0 .
$$

Combining this condition with relation (32) and taking into account the definition of the bare structure factors, yields the critical parameter $\chi_{\mathrm{c}}$

$$
\chi_{\mathrm{c}}=2 \chi_{\mathrm{PS}}-\frac{1}{\phi_{\mathrm{S}}}+\left[\left(\frac{1}{x f N \phi}+\frac{1}{\phi_{\mathrm{S}}}-2 \chi_{\mathrm{PS}}\right)\left(\frac{1}{(1-x) f N \phi}+\frac{1}{\phi_{\mathrm{S}}}-2 \chi_{\mathrm{PS}}\right)\right]^{\frac{1}{2}} .
$$

This relation expresses the dependence of $\chi_{\mathrm{c}}$ upon the monomer concentration, composition, molecular weight, and polymer-solvent interaction parameter. In the high polymer concentration limit, we obtain from relation $(38 a)$ the simplified formula

$$
\chi_{\mathrm{c}} \simeq \frac{1}{2 \phi N f x(1-x)} .
$$

This expression tells us, firstly, that the ramification chains have in effect to reduce the compatibility domain. Secondly, it shows that $\chi_{\mathrm{c}}$ (solution) $<\chi_{\mathrm{c}}($ bulk $)=2 / N f$, since $\phi<1$. 


\section{Conclusions}

We recall that the purpose of the present work was to investigate the scattering properties of ramified polymeric systems. We have considered as systems: (1) ramified polymers in a solution, (2) ramified polymer blends, and (3) ternary mixtures made of two chemically different ramified polymers immersed in a good solvent. We assumed that these ramified polymers have arbitrary topology.

We first determined the exact expression of the form factor of an ideal ramified polymer of any topology. In this expression, there appear two functions, which are the Debye function and some function depending on the topological structure of the polymer network. We studied all analytical properties of this form factor. From this latter, we extracted the exact expression of the gyration radius for any topology.

The second step was to determine the exact expression of the structure factor of ramified polymers in a solution, through the classical Zimm's formula, where there appear the form factor and the excluded volume parameter. As a typical example, we have considered star-polymers, which are networks made of chains tied together to some reticulation point. For this particular system, we have investigated the behavior of the structure factor versus the wave-vector, at fixed parameters of the problem, which are the number of chains inside the stars, the polymer concentration and the excluded volume parameter. Also, we have explored the variation of the structure factor (at a fixed wave-vector), when the number of branches is varied. We have shown, in particular, that the structure factor of polymer networks of a given topology is more important than that relative to linear chains having the same number of monomers. This occurs for all values of the wave-vector and parameters of interest. This tendency clearly shows the role of chain ramification, which leads to a reduction of the fluctuations of monomer concentration.

The third and fourth steps were the investigation of the scattering properties from ramified polymer blends and ternary mixtures, respectively. Use was made of the RPA method. The main result is that, the presence of reticulation points induces drastic changes of the scattering properties.

We note that, in any case, the structure factor of star-polymers, whatever the value of their number of branches $f$, is a monotonically decreasing function of the wave-vector. In principle, the structure factor may present a peak at a nonvanishing wave-vector only if the system exhibits a microphase separation, like diblock-copolymers, interpenetrating networks or polyelectrolytes. Now, if one is interested in the scattering-intensity from the cores of the star-polymers (viewed as colloids), this physical quantity presents at least one (principal) peak at a nonvanishing wave-vector, as experimentally demonstrated by Likos and coworkers [28] for star-homopolymers in a solution.

Finally, we emphasize that an extension to multicomponent charged ramified polymeric systems is in progress [29]. 


\section{Acknowledgements}

We are much indebted to Professors T.A.Vilgis and M.Daoud for useful correspondence. We are grateful to our referee for his critical reading of the manuscript and useful suggestions. One of us (N.G) would like to thank the Laboratoire de Physique des Polymères et Phénomènes Critiques for their kindly hospitality during his regular visit.

\section{Appendix}

This appendix is devoted to the proof of inequality (13b), according to which the structure factor of a given network is greater than that of a linear chain having the same number of monomers. To demonstrate such an inequality, it will be sufficient to show that the topological function $h_{\mathrm{G}}(u)$ is greater than its homologous $h_{\mathrm{L}}(u)$, defined by equations (10) and (12a), respectively.

Firstly, we note that the topological function $h_{\mathrm{G}}(u)$ is a monotonically decreasing function of the $u$-variable. Indeed, its first derivative

$$
h_{\mathrm{G}}^{\prime}(u)=-\sum_{\{\gamma \subset G ;|\gamma| \geqslant 2\}}|\gamma| \exp \{-|\gamma| u\}
$$

is negative for all values of $u$. In addition, this function is convex, because its second derivative

$$
h_{\mathrm{G}}^{\prime \prime}(u)=\sum_{\{\gamma \subset G ;|\gamma| \geqslant 2\}}|\gamma|^{2} \exp \{-|\gamma| u\}
$$

is positive. The function $h_{\mathrm{L}}(u)$ satisfies the same properties, since the linear network $L$ is a particular network. On the other hand, at infinity, that is $u \rightarrow \infty$, the two topological functions go to zero according to

$$
\begin{aligned}
& h_{\mathrm{G}}(u) \sim A_{\mathrm{G}} \mathrm{e}^{-2 u}, \quad(u \rightarrow \infty), \\
& h_{\mathrm{L}}(u) \sim A_{\mathrm{L}} \mathrm{e}^{-2 u}, \quad(u \rightarrow \infty),
\end{aligned}
$$

where $A_{\mathrm{G}}$ and $A_{\mathrm{L}}$ are the numbers of paths made of 2 chains inside the networks $G$ and $L$, respectively. Because of the presence of ramifications within $G$, some chains are connected at least to 2 chains. This implies that $A_{\mathrm{G}}>A_{\mathrm{L}}$. Hence, the function $h_{\mathrm{G}}(u)$ goes to zero more slowly than $h_{\mathrm{L}}(u)$.

The last step consists in making a comparison between slopes $h_{\mathrm{G}}^{\prime}(0)$ and $h_{\mathrm{L}}^{\prime}(0)$ of the two functions at the origin $u=0$. We have

$$
\begin{aligned}
& h_{\mathrm{G}}^{\prime}(0)=-\sum_{\{\gamma \subset G ;|\gamma| \geqslant 2\}}|\gamma|<0, \\
& h_{\mathrm{L}}^{\prime}(0)=-\sum_{\{\gamma \subset L ;|\gamma| \geqslant 2\}}|\gamma|<0 .
\end{aligned}
$$


Thus, $-h_{\mathrm{G}}^{\prime}(0)$ and $-h_{\mathrm{L}}^{\prime}(0)$ represent the total lengths of the connected paths inside the networks $G$ and $L$, respectively. We note that $G$ and $L$ have the same number of the connected paths $\gamma^{\prime}$ s $(|\gamma| \geqslant 2)$, that is $f(f-1) / 2$, but within $G$, there are more paths of short length than inside $L$. Consequently, we have

$$
h_{\mathrm{G}}^{\prime}(0)>h_{\mathrm{L}}^{\prime}(0) \text {. }
$$

The monotonical character of the two topological functions $h_{\mathrm{G}}(u)$ and $h_{\mathrm{L}}(u)$, the fact that $h_{\mathrm{G}}(0)=h_{\mathrm{L}}(0)$, their behaviors at infinity and inequality $(A .5)$, imply that the curve representing the function $h_{\mathrm{G}}(u)$ remains above that of $h_{\mathrm{L}}(u)$. Therefore, $h_{\mathrm{G}}(u)>h_{\mathrm{L}}(u)$.

This ends the proof of inequality (13b). 


\section{References}

1. Daoud M., Cotton J.P. // J. Phys. (Paris), 1982, No. 43, p. 531.

2. Grest G.S., Fetters L.J., Huang J.H., Richter D. // Adv. Chem. Phys., 1996, No. XCIV, p. 6.

3. Likos C.N. // Phys. Rep., 2001, No. 348, p. 267;

von Ferber C., Holovatch Yu. (Eds.) Star Polymers. // Condens. Matter Phys., 2002, vol. 5 , No. 1.

4. de Gennes P.-G. Scaling Concept in Polymer Physics. Ithaca, Cornell University Press, 1979.

5. des Cloizeaux J., Jannink G. Polymers in Solution. Oxford, Oxford University Press, 1990.

6. Pines D. Elementary Excitations in Solids. New York, Bejamin W.A., 1963.

7. Joanny J.-F., Leibler L., Ball R. // J. Chem. Phys., 1984, No. 81, p. 4640.

8. Broseta D., Leibler L., Joanny J.-F. // Macromolecules, 1987, No. 20, p. 1935.

9. Zinn-Justin J. Quantum Field Theory and Critical Phenomena. Oxford, Clarendon Press, 1989.

10. Itzykson C., Drouffe J.M. Statistical Field Theory: 1 and 2. Cambridge University Press, 1989.

11. Duplantier B. Thesis. Paris VI-University, 1982.

12. Cates M.E., Witten T.A. // Phys. Rev. A, 1987, No. 35, p. 1809.

13. Duplantier B. // J. Stat. Phys., 1989, No. 54, p. 581.

14. Schäffer L., von Ferber C., Lehr U., Duplantier B. // Nucl. Phys. B, 1992, No. 374, p. 473.

15. von Ferber C., Holovatch Yu. // Theor. Math. Physics, 1996, No. 109, p. 1274.

16. von Ferber C., Holovatch Yu. // Europhys. Lett., 1997, No. 39, p. 31.

17. Duplantier B., Ludwig A.W.W. // Phys. Rev. Lett., 1991, No. 66, p. 247.

18. Badia M., Benhamou M., Derouiche A., Bretonnet J.-L. // Colloid \& Polym. Sci., 2001, No. 279, p. 763.

19. von Ferber C., Holovatch Yu. // Phys. Rev. E, 1997, No. 56, p. 6370;

von Ferber C., Holovatch Yu. // Phys. Rev. E, 1999, No. 59, p. 6914;

von Ferber C., Holovatch Yu. // Phys. Rev. E, 2002, No. 65, p. 42801.

20. Schulte-Frohline V., Holovatch Yu., von Ferber C., Blum A. // Condens. Matter Phys., 2003, No. 6, p. 703, and references therein.

21. Zimm B. // J. Phys. Chem., 1948, No. 16, p. 1093.

22. Edwards S.F. // Proc. Phys. Soc. (London), 1965, No. 85, p. 613.

23. Guinier A., Fournet G. Small Angle Scattering of X-Rays, John Wiley and Sons. New York, 1955.

24. Yamakawa H. Modern Theory of Polymer Solutions, formula (9.69). New York, Harper \& Row, 1971.

25. Vilgis T.A., Benmouna M., Benoit H. // Macromolecules, 1991, No. 24, p. 4481.

26. Benmouna M., Vilgis T.A. // Macromolecules, 1991, No. 24, p. 3866.

27. Flory P.J. Principles of Polymer Chemistry. Ithaca, Cornell University Press, 1953.

28. Likos C.N., Löwen H., Watzlawek M., Abbas B., Jucknischke O., Allgaier J., Richter D. // Phys. Rev. Lett., 1998, No. 80, p. 4450.

29. Ghaouar N., Benhamou M., Benmouna M., Gharbi A., (in preparation). 


\title{
Розсіяння з розгалужених полімерних систем
}

\author{
М.Бенхамоу ${ }^{1}$, Н.Жаоуар ${ }^{1,2}$, А.Жарбі ${ }^{2}$, М.Бенмоуна ${ }^{3}$
}

1 Лабораторія фізики і критичних явищ, факультет природничих наук Бен М'сік, В.Р. 7955, Касабланка, Марокко

2 Лабораторія фізики м'якої речовини, факультет природничих наук Тунісу, Туніс

3 Інститут полімерних досліджень, товариство Макса Планка 3148, D-55021, Майнц, Німеччина

Отримано 4 серпня 2003 р., в остаточному вигляді - 30 січня $2004 \mathrm{p}$.

Тут представлені кількісні дослідження властивостей розсіяння 3 розгалужених полімерних систем довільної топології. Ми розглядаємо три типи систем, а саме: розгалужені полімери в розчині, розгалужені полімерні суміші, потрійні суміші, що складаються з двох розгалужених полімерів різної хімічної природи, занурених в добрий розчинник. Для досягнення мети застосовується наближення хаотичних фаз. Спершу ми визначаємо точний вираз для формфактора ідеального розгалуженого полімера довільної топології, на основі якого потім отримуємо точний вираз для радіуса гірації. Використовуючи класичні формули Зімма і точний формфактор, ми визначаємо всі властивості розсіяння вищезгаданих трьох типів розгалужених полімерних систем. Основним висновком є, що розгалуження ланцюжків індукує значні зміни у властивостях розсіяння.

Ключові слова: колоїди, макромолекулярні і полімерні розчини, полімерні розплави, набухання, розчинність, сегрегація, фазове відокремлення змішування

PACS: 82.70.Dd, 61.25. Hq, 64.75. $+\mathrm{g}$ 